\title{
Research paper on IOT based Air and Sound Pollution Monitoring System
}

\author{
Lalit Mohan Joshi \\ PhD Research Scholar \\ B.T.K.I.T. Dwarahat (India)
}

\begin{abstract}
In infrastructure and industrial plants the rapid growth creating environmental issues like pollution (Air, Water, Noise), climate change, malfunctioning and has greatly consequence for the requirement of an, operationally adaptable, efficient, cheap and smart monitoring systems. In this context where combination of many challenges of computer science, wireless communication and electronics; the Smart Sensor Networks are an emerging field of research. In this paper a solution to monitor the air and noise pollution levels in industrial environment or by using wireless embedded computing system a particular area of interest is proposed. The technology like Internet of Things (IoT) is included in the form of solution which is outcome of merged field of computer science and electronics. For monitoring the fluctuation of parameters like noise and air pollution levels from their normal levels in this case the sensing devices are connected to the embedded computing system. For the requirement of continuous monitoring, controlling and behavior analysis this model is adaptable and distributive for any infrastructural environment. The working appearance of the proposed model is evaluated using prototype implementation, consisting of AVR UNO board, sensor devices and MATLAB with AVR hardware support package. For two or three parameters like noise, $\mathrm{CO}$ and radiation levels the implementation is tested with respect to the normal behavior levels or given specifications which provide a monitoring over the pollution control to make the environment smart and ecofriendly. The basic mission of the Air Quality Planning and Standards is to preserve quality of air .The level of pollution in air can be measured by measuring the pollutants such as humidity level, temperature level, dust level, CO level, smoke level etc present in the air of that area. Here we propose an air quality pollution monitoring system that allows us to monitor and check live air quality in a particular areas through IoT.
\end{abstract}

With the fast growing technology, it would be great to get to know about our surrounding weather parameters in this widely connected environment of internet when one can easily access the rarest and the farthest information at one's own fingertips. This project is based on IoT (Internet of Things), which is an emerging field in which all the devices are connected to a channel made by self (private channel). The channel is used to view the weather parameters with unique API key of channel of a particular user. Every channel has both Read and Write API keys to get the access. Wi-Fi module, temperature, humidity, gas, and dust sensors are interfaced with the Xmega 2560. The user is prompted to provide the API key of channel. ESP8266-01 reads the key and sends it to the Xmega 2560. If the key is matched, then the data transmission can be carried out between the channel and the microcontroller. The module is connected to the $\mathrm{Wi}-\mathrm{Fi}$ through some AT Commands.

\section{Keywords}

Xmega, Xbee Nodes,

\section{INTRODUCTION}

To control and monitor of different activities focused by Present innovations in technology. To reach the human needs these are increasingly emerging. Most of this technology is focused on efficient monitoring and controlling different activities. To monitor and assess the conditions in case of exceeding the prescribed level of parameters (e.g., noise, $\mathrm{CO}$ and radiation levels) an efficient environmental monitoring system is needed. In an environment when an object equipped with sensor devices, then in this case microcontroller and various software applications becomes a self-defending Selfmonitoring and self-controlling environment and it is also called as smart environment.

In such environment when LED alerts automatically or some event occurs the alarm. Smart Environmental Monitoring System monitor and control the ecological changes on animals, plants and human beings on the basis effects due to environmental changes. By using embedded intelligence into the environment makes the environment interactive with other objectives, this is one of the application that smart environment targets Human needs demands different types of monitoring systems these are depends on the type of data gathered by the sensor devices. Event Detection based and Spatial Process Estimation are the two categories to which applications are classified. Initially the sensor devices are deployed in environment to detect the parameters (e.g., noise, $\mathrm{CO}$ and radiation levels etc.) while the data acquisition, computation and controlling action (e.g., with respect to the specified levels, the variations in the noise and CO levels ). To predict the behavior of a particular area of interest and to collect the data, the Sensor devices are placed at different locations. The main goal of the this paper is to design and implement an adequate monitoring system through which the needed parameters are monitored and controlled remotely by using internet and the data collected from the sensors are stored in the cloud and on the web browser to project the estimated trend.

A solution for monitoring the noise and CO levels i.e., any parameter value crossing its threshold value ranges, for example $\mathrm{CO}$ levels in air in a particular area exceeding the normal levels etc., in the environment using wireless embedded computing system is proposed in this paper. The solution also provides an intelligent remote monitoring for a particular area of interest. In this paper $i$ also describe a trending results of sensed or collected data with respect to the ordinary as well as specified ranges of particular parameters. The embedded system enables the user to remotely access the various parameters and store the data in cloud and This system is an integration of sensor devices with wireless communication. The quality of air is important for the survival of living beings. It is necessary to monitor air quality 
and keep it under control for a better future and healthy living for all. The basic mission of this project is to preserve and improve the quality of air. Compared to natural sources, man's activities produce a much smaller amount of global pollution. Any substantial change in the nature or contents of the atmosphere has a direct consequence on how well the atmosphere performs these tasks. Atmospheric problems are made worse by weather conditions, so we should know about it correctly. Figure 1.1 Global Emissions From Natural \& Man Made Sources The level of pollution in air can be measured by measuring the pollutants such as humidity level, temperature level, dust level, $\mathrm{CO}$ level, smoke level etc. present in the air of that area. Here we propose an air quality pollution monitoring system that allows us to monitor and check live air quality in a particular area through IoT. The sensors interact with microcontroller which processes this data and transmits it over internet. This allows authorities to monitor air pollution in different areas and take action to control the issue. Here we propose an air quality pollution monitoring system that allows us to monitor and check live air quality in particular areas through IoT. To sense presence of harmful gases/compounds in the air and constantly transmit this data to microcontroller; Air sensors are used by the system. The interaction of sensors with microcontroller processes this data as well as transmits it over internet.

This project aims at benefitting the modern society aided with the constantly increasing use of internet. IoT (Internet of Things) IoT is simply the network of interconnected things/devices which are embedded with sensors, software, network connectivity and necessary electronics that enables them to collect and exchange data making them responsive. Internet of Things is essentially an architectural framework more than a concept in which data exchange and integration allowed between the computer systems and physical world over consisting network infrastructure which exists in real world. With the help of sensors and actuators when IoT is augmented, the technology becomes an instance of the more normal class of cyber- physical systems, which also circumscribe technologies such as an intelligent transportation, smart grids, smart homes, and smart cities. Through its embedded computing system each thing is uniquely classifiable but within the existing internet infrastructure it is able to interoperate. With the help of various existing technologies the devices collect useful data and then autonomously flow the data between other devices. Current project and such other projects like smart homes such as control and automation of heating, ventilation, air conditioning etc. uses Wi-Fi for remote monitoring. This project would also work in taking care of home and atmosphere as we could monitor the parameters from anywhere under the Wi-Fi access. This allows authorities to monitor air pollution in different areas and take action to control the issue. Main motive of this project is to prevent the harmful effects of pollutants present in air so that healthy surroundings can be maintained by data analysis of stored data in IoT cloud.

\subsection{Existing System model}

In today's world many pollution monitoring systems are designed by considering different environmental parameters. Existing system model is presented in figure.1 uses Zigbee based wireless sensor networks to monitor physical and environmental conditions with thousands of application in different fields. The sensor nodes directly communicated with the moving nodes deployed on the object of interest which avoided the use of complex routing algorithm but local computations are very minimal. RFID is a means of storing and retrieving data through electromagnetic transmission to an RF compatible integrated circuit. It is basically used to track and label items in supermarkets and manufactories. There are two main components of RFID systems: tags and readers. A tag has an unique identification (ID) number and a memory which is used to store additional data such as manufacturer, product type, and environmental factors such as temperature, humidity, etc. Through Wireless Communication the reader is capable to write and/or read data to tags., In need of identification or tracking, tags are embedded or attached into objects in a typical RFID application. RFID tags can be classified into three major categories by their power source: active tags, passive tags, and semi passive (semi-active) tags are embedded or attached into objects in a typical RFID application. 


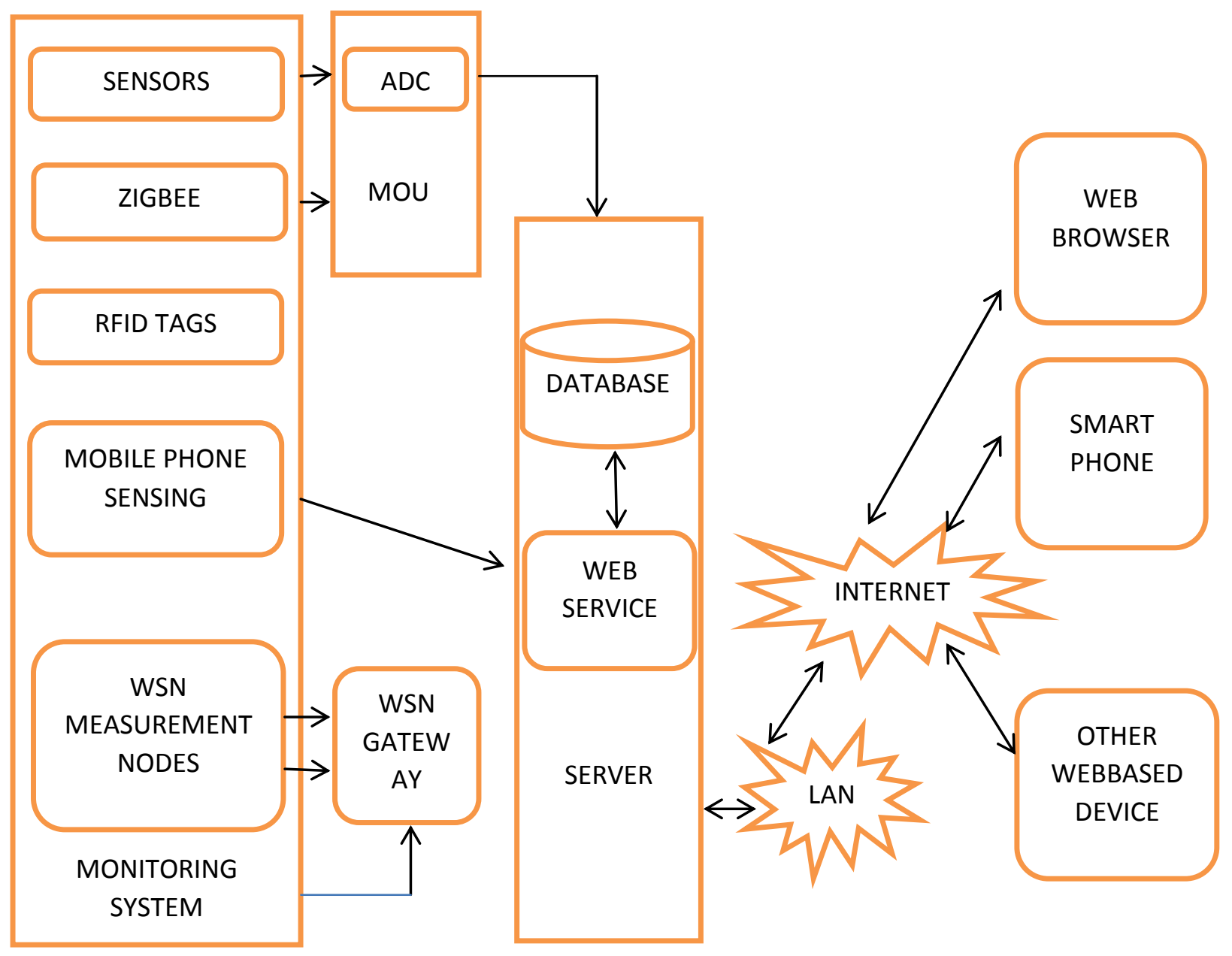

Fig 1: Existing System Model

Mobile phones or smart phones those are cap abled and in built with sensors are applicable for impact on social including how technology of mobile has to be used for to protect environmental, sensing and to influence just-in-time information to create movements and actions ecofriendly. Mobile phone sensors were deployed and used on urban areas for monitoring and it was categorized into two major classes, participatory sensing where user is directly involved and opportunistic sensing where user is not involved, but its limitation includes power and static information processing or mobility restrictions. A Wireless Sensor Network inbuilt of many wireless sensors those are inexpensive, which are able to collect, store, process an environmental information, and to communicate with nodes those are neighbors to each other. Previously, sensors are connected by wire lines. The access method of WSN gateway node is convenient because data can be received from a WSN via the gateway at any time and any place. In charge of node authentication, message buffering, the gateway works as the network coordinator where you can collect, process, analyze, and present your measurement data. Wireless sensor network management model consists of end device, router, gateway node and management monitoring center. To collect wireless sensor network data, and to forward them to parent node, Hence end device is trustworthy then data are forward to gateway node from parent node directly or with the help of router. Gateway node extracts data after getting data from wireless sensor network, then analyzes and packaged them into Ethernet format data, forward them to the server. A server is an occurrence of a computer program that is used to accept then reply to another program request; called as a client. So we could say that to run the server software any device could be considered a server. To manage network resources Servers are used. In the servers, the services or information are provided through the Internet those are connected through LAN and made available for users via smart phones, web browser or other web browser devices to make the system more intelligent, adaptable and efficient.

\subsection{Previous Work}

To make the environment smart in that area in that case some of the research works completed to monitor the parameters of pollution in a particular area of interest, where a large kind of techniques as well as methods were used in the past. Smart Environment Monitoring using Wireless Sensor networks - In this work they are mainly focus on the making the city environment smart, by deploying wireless sensor networks in all over the city and moving public transportation system buses and cars. By accessing all the sensor networks, environmental behaviors are collected as a streaming data base to identify the environmental conditions. This methodology gives the monitoring data from stationary nodes deployed in city to the mobile nodes on public transportation buses and cars.

Toward a Green Campus with the Internet of Things - the Application of Lab Management this research work adopts the concept of "Internet of Things" and implements an idea of 
energy-saving by proper management of computers and air conditioners. The architecture and the prototype of the system is explained.

Here the objects of Internet of Things are computers and air conditioners. IOT-Based and WSN Smart Homes and Smart Buildings extension of them -This work mainly desire to design and develop predictable, effective, durable, costeffective, concurrent-time and realistic wellness sensor networks for smart home systems. In wireless networking technologies where the sensor and actuator nodes are deployed into the home environment. Real-time data generated by these nodes and they all are related to the object manage and displacement inside the home.

\subsection{Proposed model}

The proposed embedded device is for monitoring noise and $\mathrm{CO}$ levels in the atmosphere to make the environment intelligent or interactive with the objects through wireless communication. The proposed model is shown in figure 2 which is more adaptable and distributive in nature to monitor the environmental parameters. The proposed architecture is discussed in a 4- tier model with the functions of each individual modules developed for noise and air pollution monitoring. The proposed model consists of 4 -tiers. The tier 1 is the environment, sensor devices in tier 2 , sensor data acquisition and decision making in tier 3 and intelligent environment in tier 4.The proposed architecture is shown in figure 2 .

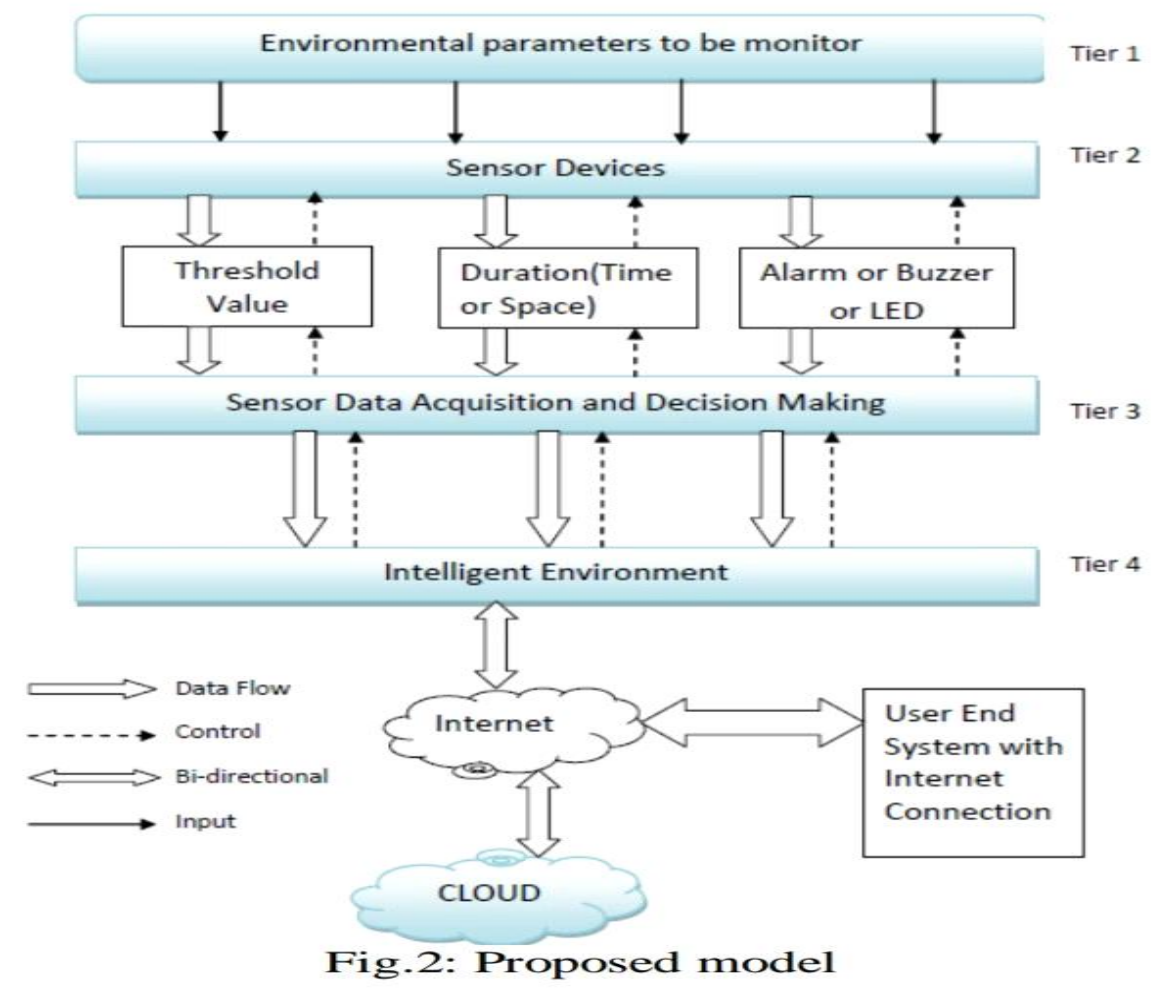

Here, the tier 1 provides information about the parameters under the region which is to be monitored for noise and air pollution control. Tier 2 deals with the sensor devices with suitable characteristics, features and each of these sensor devices are operated and controlled based on their sensitivity as well as the range of sensing. In between tier 2 and tier 3 necessary sensing and controlling actions will be taken depending upon the conditions, like fixing the threshold value, periodicity of sensing, messages (alarm or buzzer or LED) etc. Based on the data analysis performed in between tier 2 and tier 3 and also from previous experiences the parameter threshold values during critical situations or normal working conditions are determined. Tier 3 describes about the data acquisition from sensor devices and also includes the decision making. Which specify the condition the data is representing which parameter. In the proposed model tier 4 deals with the intelligent environment. Which means it will identify the variations in the sensor data and fix the threshold value depending on the identified level of $\mathrm{CO}$ or noise levels. In this tier sensed data will be processed, stored in the cloud i.e.in to the Google spread sheets and also it will show a trend of the sensed parameters with respect to the specified values. The end users can browse the data using mobile phones, PCs etc.

Based on the framework shown in figure 2, we have identified a suitable implementation model that consists of different sensor devices and other modules, their functionalities are shown in figure 3.In this implementation model we used AVR UNO board with Wi-Fi module is as embedded device for sensing and storing the data in cloud. AVR UNO board consist of analog input pins (A0-A5), digital output pins (D0D13), inbuilt ADC and Wi-Fi module connects the embedded device to internet. Sensors are connected to AVR UNO board for monitoring, ADC will convert the corresponding sensor reading to its digital value and from that value the corresponding environmental parameter will be evaluated. The Wi-Fi connection has to be established to transfer sensors data to end user and also send it to the cloud storage for future usage. Before sending the sensed data to cloud, the data will be processed in MATLAB for analyze and visualize data to end user. The data analysis in MATLAB makes easier to us to set threshold level and to perform necessary controlling actions 


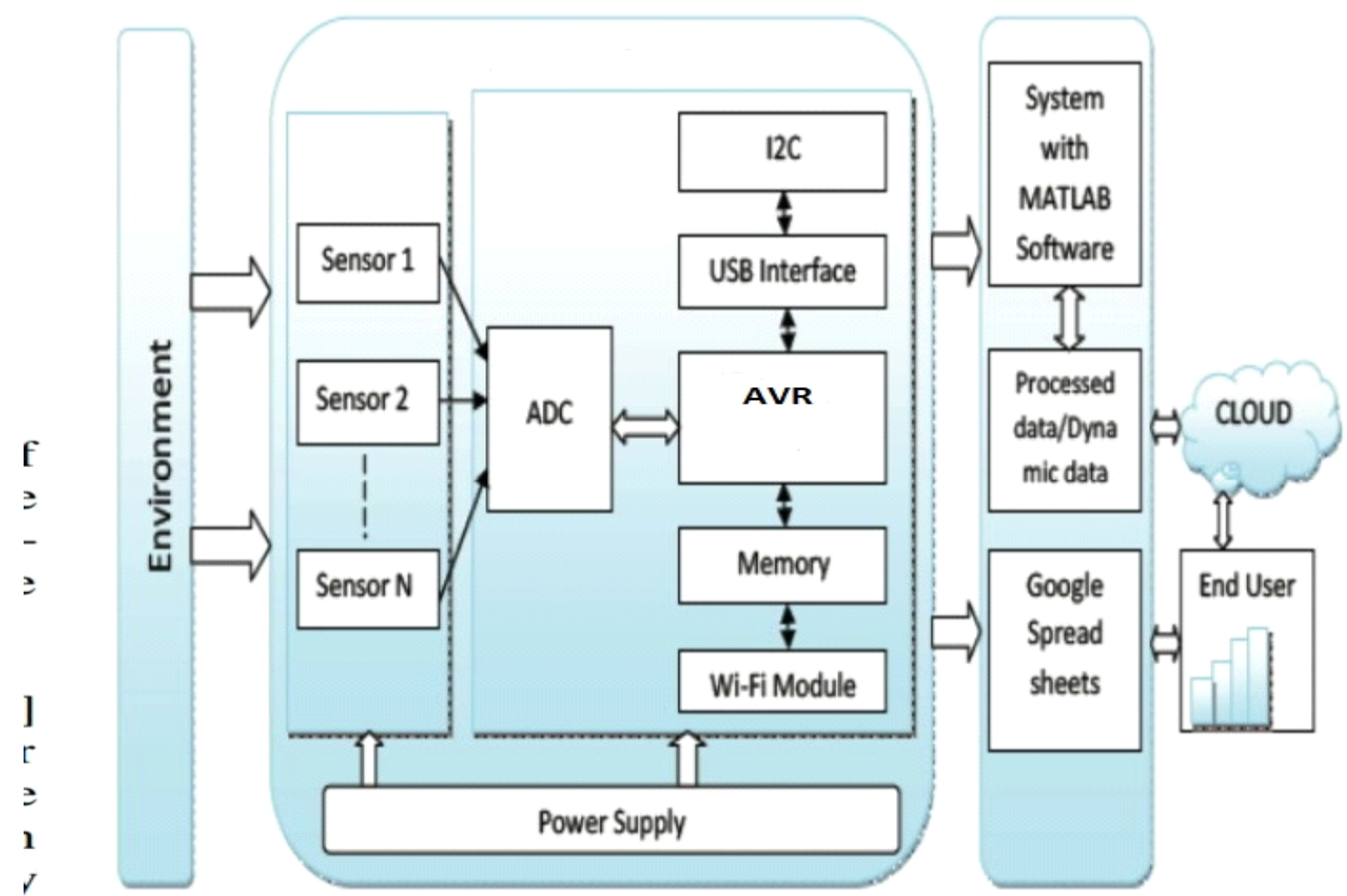

Fig. 3: Schematic diagram of implementation model

An embedded system designed for environmental monitoring and its components are shown in figure 4.The embedded device is placed in particular area for testing purpose. The sound sensor detects sound intensity levels in that area and Carbon Monoxide (CO) sensor MQ-9 will record the air quality in that region, if the threshold limit is crossed the corresponding controlling action will be taken (like issuing message alarm or buzzer or LED blink).All the sensor devices are connected to internet through Wi-Fi module.

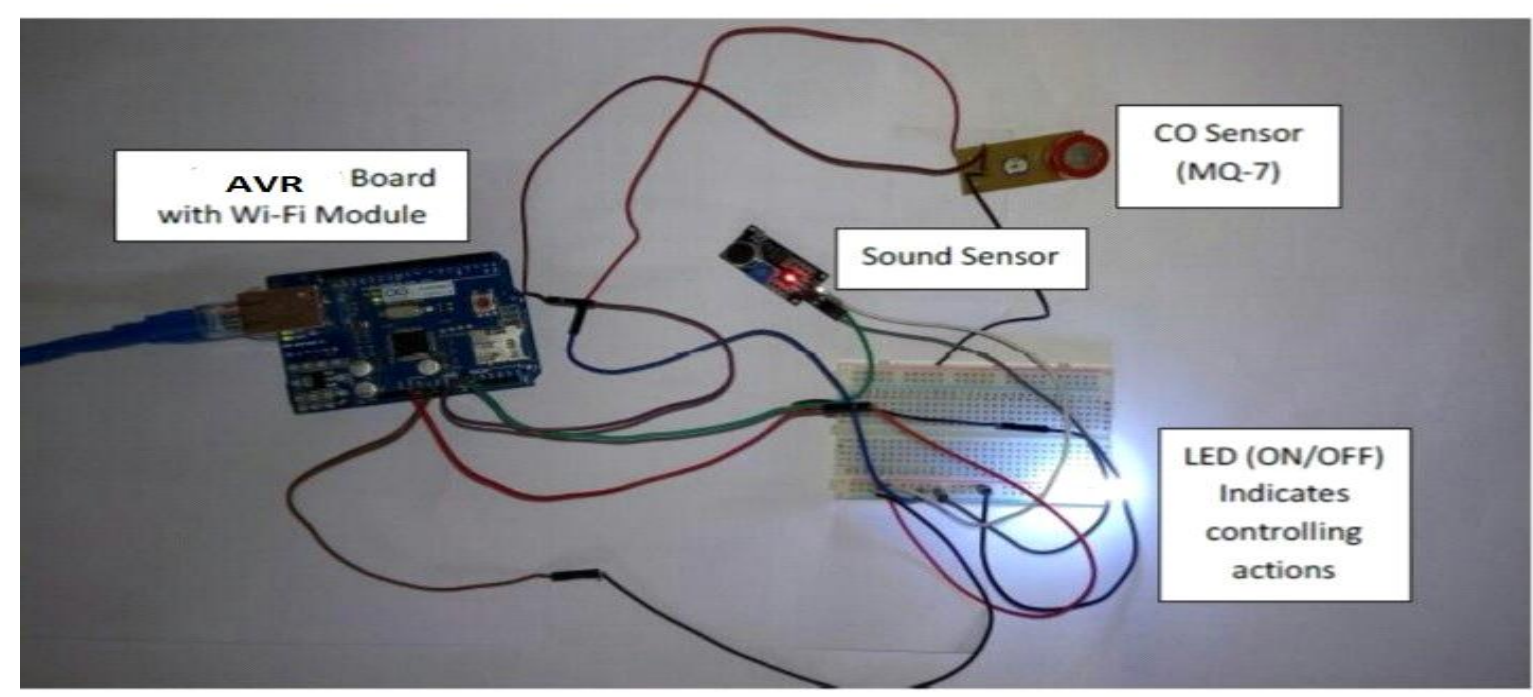

Fig. 4: Noise and air pollution monitoring embedded system with its components

It shows the embedded system with its components for reading and to store the pollution parameters in cloud. After successful completion of sensing, the data will be processed and stored in database for future reference. After completing the analysis on data the threshold values will be set for controlling purpose.
CO levels in air at regular time intervals. All the above information will be stored in the cloud, so that we can provide trending of noise intensity and $\mathrm{CO}$ levels in a particular area at any point of time.

After sensing the data from different sensor devices, which are placed in particular area of interest. The sensed data will be automatically sent to the web server, when a proper 
connection is established with sever device. The figure 5 shows the web server page which will allow us to monitor and control the system. By entering IP address of server which is placed for monitoring we will get the corresponding web page. The web page gives the information about the intensity of sound and the $\mathrm{CO}$ level variations in that particular region, where the embedded monitoring system is placed.

The sensed data will be stored in cloud (Google Spread Sheets). The data stored in cloud can be used for the analysis of the parameter and continuous monitoring purpose. The figure 6 shows the noise intensity levels and $\mathrm{CO}$ levels in air at regular time intervals. All the above information will be stored in the cloud, so that we can provide trending of noise intensity and $\mathrm{CO}$ levels in a particular area at any point of time.

\subsection{Working of the project}

In view of the daily chores of a household the monitoring of real time parameters is highly beneficial for the sustenance of an organized and well informed standard of living. The parameters we are dealing here are different gases, dust, room temperatures and humidity present required for an ambient living. The respective sensors are being utilized for acquisition of the required data to be monitored as shown in Figures in next. DHT11 sensor has been used to monitor the room temperature and pressure. The sensor is soldered onto a PCB with a $3.3 \mathrm{~V}$ regulator, I2C level shifter and pull-up resistors on the $\mathrm{I} 2 \mathrm{C}$ pins. It is a 4 pins sensor of which 2 pins uses I2C protocol (SCL and SDA) which are connected to the respective I2C protocol supported pins on the Xmega 2560. It is based on the piezo resistive technology. The parameters are then send to the microcontroller MQ-2 sensor is a gas sensor which can detect the presence of combustible gases such as ibutane, LPG, hydrogen \& methane. In this project, it is used mainly for detecting household LPG. The ionized constituents are detected by the sensing element, which creates a potential difference thus giving output in the form of current. The concentration of the gas detected is then send to the Xmega 2560. It has both the analog and the digital output but here we use the analog output which is connected to the analog pin of the microcontroller. DHT11 sensor is used for measuring humidity and temperature of the surroundings but, here I had used it for measuring only the humidity. The sensor provides fully calibrated digital outputs for the measurements of the parameters. It sends a data of 40 bits both for the temperature and humidity which also includes the checksum byte (bit error check). It operates at a voltage of $+5 \mathrm{v}$ and gives the digital output connected to any of the digital pin of the microcontroller.

The MQ-135 gas sensor senses the gases like CO2, ammonia nitrogen, oxygen, alcohols, aromatic compounds, sulfide and smoke. The operating voltage of this gas sensor is from $2.5 \mathrm{~V}$ to $5.0 \mathrm{~V}$. In the atmosphere we can find polluting gases, but the conductivity of gas sensor increases as the concentration of polluting gas increases. MQ-135 gas sensor can be implementation to detect the smoke, benzene, steam and other harmful gases. It has potential to detect different harmful gases. The MQ-135 gas sensor is low cost to purchase. ESP8266 module is a Wi-Fi module, which the backbone of this project. Here it is used for connecting the microcontroller to an access point (Wi-Fi). This module has inbuilt set of Attention Commands which are required to configure the module. Firstly we flash the ESP8266 module using the software then using the Attention Commands it is set in the Wi-Fi mode and then it is connected to a mobile hotspot or a $\mathrm{Wi}-\mathrm{Fi}$, which finally connects our microcontroller to the $\mathrm{Wi}$ Fi.

We create a channel (private) to view the changes in the parameters. The data is displayed graphically on the channel. One can get the access to the channel by getting the user ID and the data is transmitted to the channel by using the write API key provided by the channel, which enables our microcontroller to send the data. It requires a time of $15 \mathrm{~ms}$ to update the data. Xmega $2560 \mathrm{UNO}$ is one of the varieties of Xmega 2560 board microcontroller based on Xmega 2560, which takes input from the sensors and is connected to the Wi-Fi with the help of the Wi-Fi module which enable it to transmit data to the channel. The whole processing required is done by the processor in it.

Starting with this project, first of all we flash the memory of the Wi-Fi module (ESP8266) to avoid any garbage values in our readings, then moving on to the next step we use some AT commands to set the module in the Wi-Fi mode and search for the available access points and then connect to any of them. If the module gets connected, it is well and good otherwise go back to the basic AT Commands then retry to connect which connects our microcontroller to the $\mathrm{Wi}-\mathrm{Fi}$. Then the next step is for taking inputs from the respective sensors in the microcontroller, now after obtaining values from the sensors, we need to convert the 5 volt logic of Xmega 2560 to the 3.3 volt logic as the Wi-Fi module works in 3.3 volt logic, after doing that use the channel API key to transmit the data/ input from the sensor to the channel and display them graphically on the space provided by the channel and for more understanding the whole process has been depicted.

In this the components used in the project are represented in the form of blocks and shows how we carried out our work. First of all using all the 4 types of sensors we collected data then this data is latched in the microcontroller, then after performing all the basic requirements required by the Wi-Fi module, we connect our microcontroller to the access point and then finally we upload the data to the channel. 


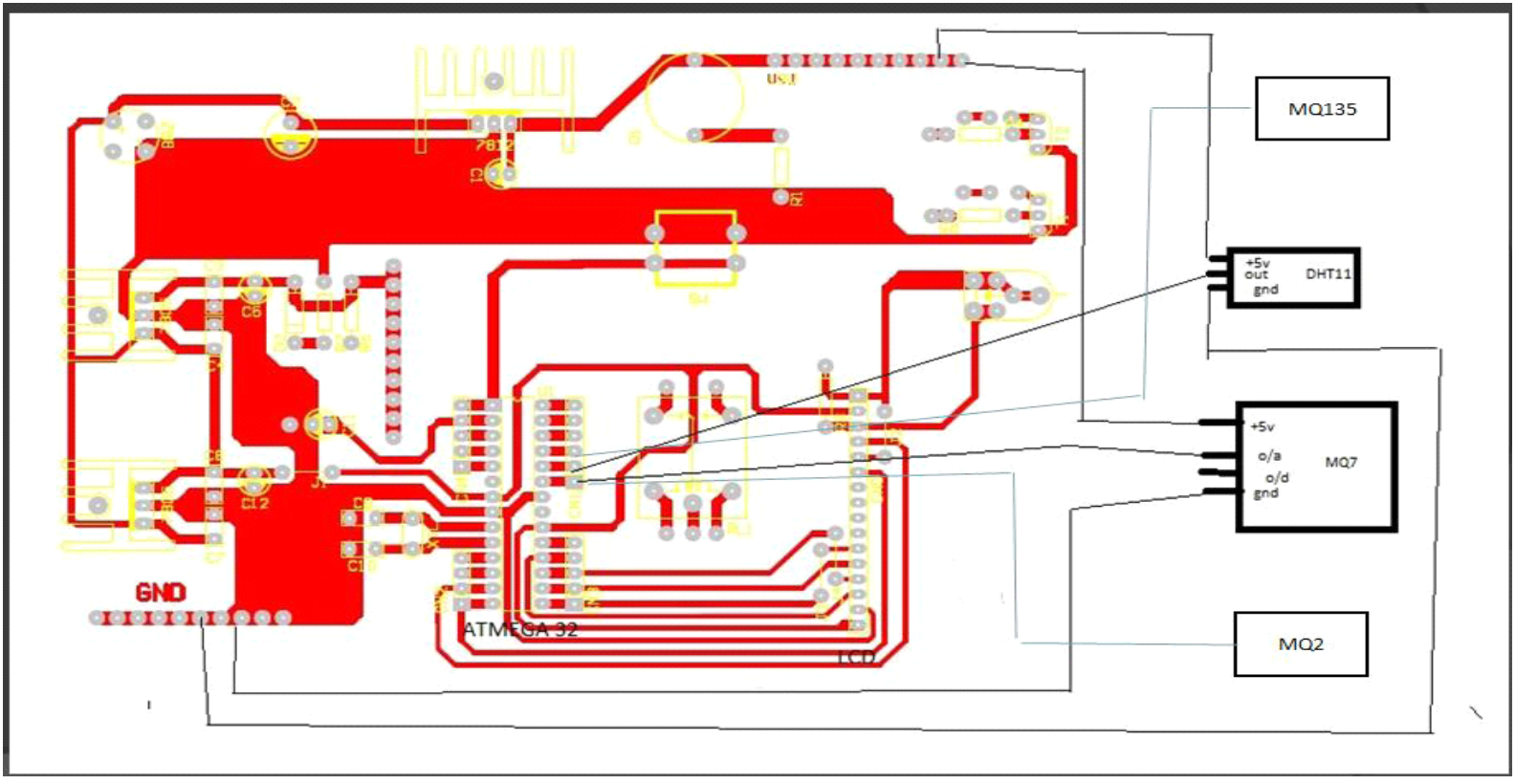

Fig 5: Block Diagram of the Project

\section{COMPONENT}

2.1 Dust Sensor (MQ135)

2.2 CO Gas Sensor (MQ7)

2.3 Smoke Sensor (MQ2)

2.4 Temperature Sensor (DHT11)

2.5 Xmega 2560 Microcontroller

2.6 Wi-Fi Modem(ESP8266)

2.7 LCD Display

2.8 LED's

2.9 Rectifier

2.10 Resistors

2.11 Capacitors

2.12 Regulator (7805,7809, LD33)

2.13 PCB Board

2.14 Xmega 2560 Uno

2.15 Battery.

\section{COMPONENT REQUIRED}

\subsection{Xmega 2560}

XMEGA 2560 is an open-source physical computing platform based on a simple microcontroller board, and a development environment for writing software for it. The Xmega 2560 Uno is a variety of Xmega 2560 board based on the Xmega 2560. It has 14 digital input/output pins (of which 6 can be used as PWM outputs), 6 analog inputs, a $16 \mathrm{MHz}$ ceramic resonator, a USB connection, a power jack, an ICSP header, and a reset button. The Xmega 2560 programming language is an implementation of wiring, a similar physical computing platform, which is based on the Processing multimedia programming environment. The microcontrollers inserted in the Xmega 2560 board can be easily programmed by imply uploading the program using IDE software. Xmega 2560 (sold as Genuine outside of the U.S. and U.K due to a trademark dispute) is a hardware and software company, project, and user community that designs and manufactures computer hardware, open-source software, and microcontroller-based kits for building digital devices and interactive objects that can sense and control physical devices. The gas sensor module consists of a steel exoskeleton under which a sensing element is housed. This sensing element is subjected to current through connecting leads. This current is known as heating current through it, the gases coming close to the sensing element get ionized and are absorbed by the sensing element. This changes the resistance of the sensing element which alters the value of the current going out of it. This is one of the main components of this project. It offers $23 \mathrm{i} / \mathrm{o}$ pins and a $16 \mathrm{mhz}$ crystal Oscillator is used to provide timing /clock reference.

\section{FEATURES OF XMEGA 2560 UNO XMEGA 2560:-}

Microcontroller: Xmega 2560

Operating Voltage: $5 \mathrm{~V}$

Input Voltage: 7-12V

Digital I/O Pins: 14 (of which 6 provided PWM output))

Analog Input Pins: 6

DC Current: $40 \mathrm{~mA}$

DC Current: 50mA

Flash Memory: 32KB

SRAM: $2 \mathrm{~KB}$

EEPROM: $1 \mathrm{~KB}$

Clock Speed: $16 \mathrm{MHz}$

\subsection{Regulator}

Voltage regulator IC's are the IC's that are used to regulate voltage. IC 7805 is a 5V Voltage Regulator that restricts the voltage output to $5 \mathrm{~V}$ and draws $5 \mathrm{~V}$ regulated power supply. 


\subsection{Mq-7 Gas Sensor}

This semiconductor gas sensor detects the presence of Carbon Monoxide at concentrations from 10 to $10,000 \mathrm{ppm}$. The sensor's simple analog voltage interface requires only one analog input pin from your microcontroller.

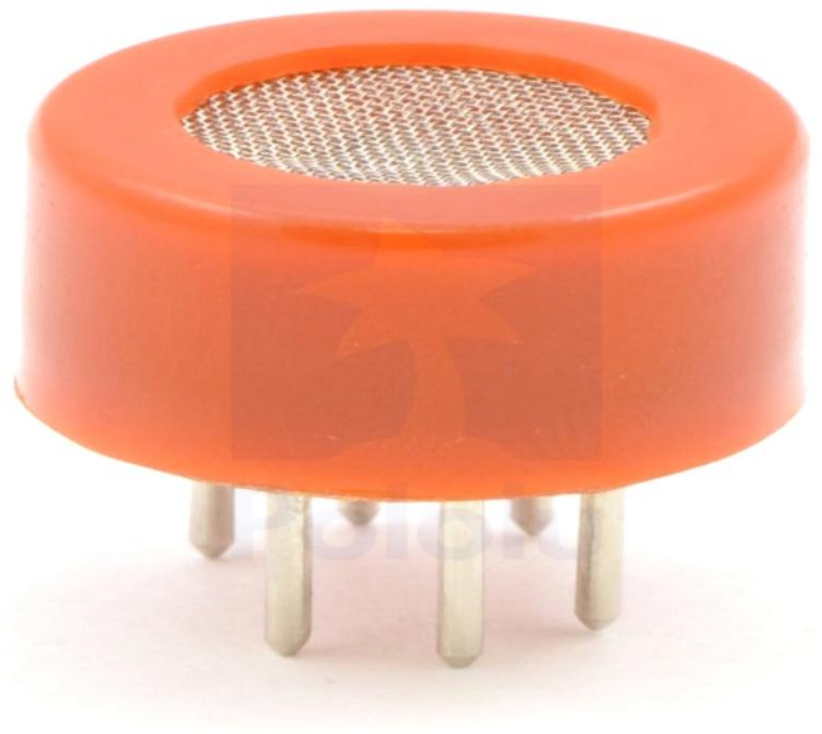

Fig 6: MQ-7Gas Sensor

This Carbon Monoxide (CO) gas sensor detects the concentrations of $\mathrm{CO}$ in the air and outputs its reading as an analog voltage. The sensor can measure concentrations of 10 to $10,000 \mathrm{ppm}$. The sensor can operate at temperatures from 10 to $50^{\circ} \mathrm{C}$ and consumes less than $150 \mathrm{~mA}$ at $5 \mathrm{~V}$.

\subsection{Pin Connection}

Connecting five volts across the heating $(\mathrm{H})$ pins keeps the sensor hot enough to function correctly. Connecting five volts at either the A or B pins causes the sensor to emit an analog voltage on the other pins. A resistive load between the output pins and ground sets the sensitivity of the detector. The resistive load should be calibrated for your particular application. This is a simple-to-use Carbon Monoxide (CO) sensor, suitable for sensing $\mathrm{CO}$ concentrations in the air. The MQ-7 can detect CO-gas concentrations anywhere from 20 to 2000ppm. This sensor has a high sensitivity and fast response time. The sensor's output is an analog resistance. The drive circuit is very simple; all you need to do is power the heater coil with $5 \mathrm{~V}$, add a load resistance, and connect the output to an ADC.

\section{ADVANTAGES OF THE PROJECT}

- Sensors are easily available.

- Interface any number of sensors to know detail content of all gases present in air.

- Detecting a wide range of gases, including $\mathrm{CO}$, MH4, alcohol, smoke etc.

- $\quad$ Simple, compact \& Easy to handle.

- $\quad$ Sensors have long life time $\&$ less cost.

- $\quad$ Simple Drive circuit.

- System is Real time.

- Operating voltage : 5 volt, $-20^{\circ} \mathrm{C}$ to $+50^{\circ} \mathrm{C}$
- Quality of air can be checked indoor as well as outdoor.

- Visual output..

- Continuous update of change in percentage of quality.

\section{TECHNICAL DETAILS}

- Low cost

- $\quad$ to $5 \mathrm{~V}$ power and $\mathrm{I} / \mathrm{O}$

- $\quad 2.5 \mathrm{~mA} \max$ current use during conversion (while requesting data)

- Good for $20-80 \%$ humidity readings with $5 \%$ accuracy

- Good for $0-50^{\circ} \mathrm{C}$ temperature readings $\pm 2^{\circ} \mathrm{C}$ accuracy

- No more than $1 \mathrm{~Hz}$ sampling rate (once every second)

- $\quad$ Body size $15.5 \mathrm{~mm}$ x $12 \mathrm{~mm}$ x $5.5 \mathrm{~mm}$

- $\quad$ pins with $0.1 "$ spacing

\subsection{Hardware Connections}

- $\quad$ DTH11 To Xmega2560

- $\quad$ Vcc--- 5v

- $\quad$ GND --- GND

- Data Pin --- pin of ATMEL 89S52

The main thing to remember is, that this device works with $3.3 \mathrm{~V}$; even the RX and TX pins. Xmega 2560 or many USB to serial converters work with $5 \mathrm{~V}$. The solution for this project is in the next step. 


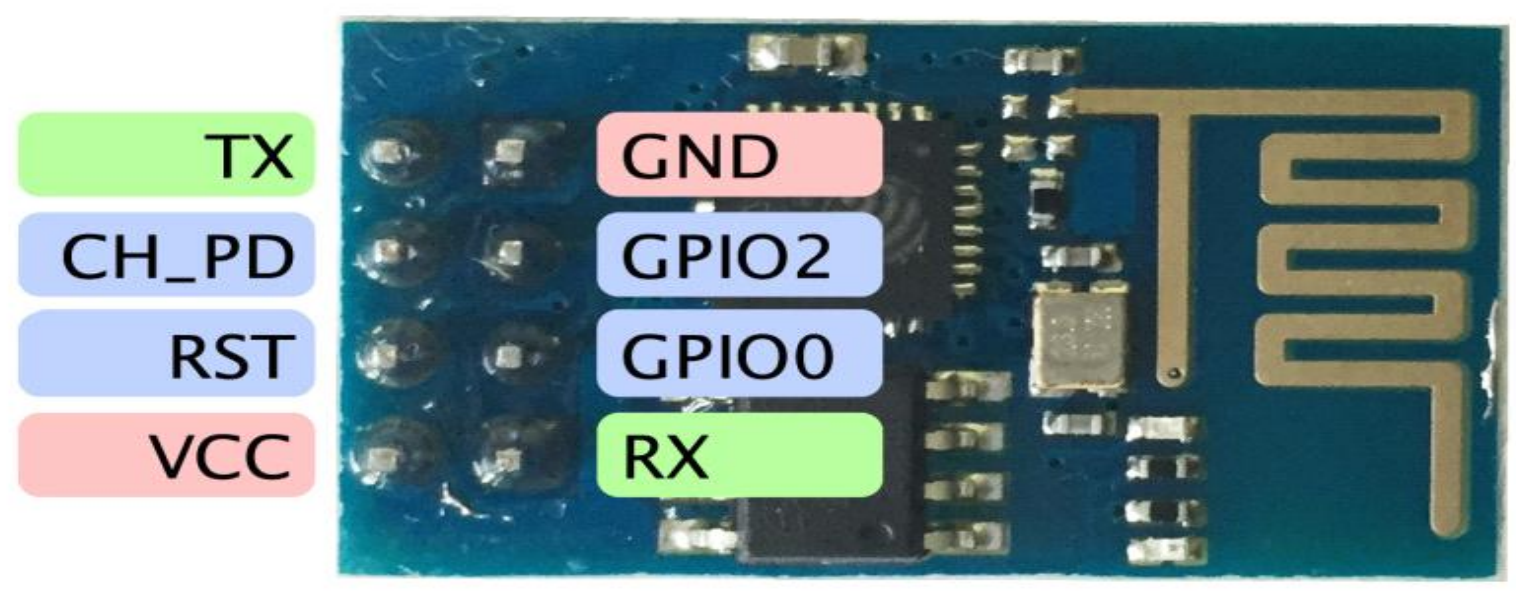

Fig 7: Hardware Connection

\subsection{Features}

- $\quad 802.11 \mathrm{~b} / \mathrm{g} / \mathrm{n}$

- Wi-Fi Direct (P2P), soft-AP

- Integrated TCP/IP protocol stack

- Integrated TR switch, balun, LNA, power amplifier and matching network

- Integrated PLLs, regulators, DCXO and power management units

- $\quad+19.5 \mathrm{dBm}$ output power in $802.11 \mathrm{~b}$ mode

- Power down leakage current of $<10 \mathrm{uA}$

- $1 \mathrm{MB}$ Flash Memory

- Integrated low power 32-bit CPU could be used as application processor

- $\quad$ SDIO $1.1 / 2.0$, SPI, UART

- $\quad$ STBC, $1 \times 1$ MIMO, $2 \times 1$ MIMO
- A-MPDU \& A-MSDU aggregation \& $0.4 \mathrm{~ms}$ guard interval

- Wake up and transmit packets in $<2 \mathrm{~ms}$

- $\quad$ Standby power consumption of $<1.0 \mathrm{~mW}$ (DTIM).

\section{SOUND SENSOR MODULE}

- A mic used to capture the sound signal.

- Sound sensitive material which converts sound energy in to electrical energy

- An amplifier used to amplify the PM358 to amplify electrical signal produced.

\subsection{Features}

- Easy to use sound sensor module

- Provide analog or digital output signal.

\subsection{Specifications}

- Operating voltage range $: 4-12 \mathrm{v}$

- Operating current $(\mathrm{vcc}=5 \mathrm{v}): 4-8 \mathrm{~mA}$

- Voltage gain 26dB

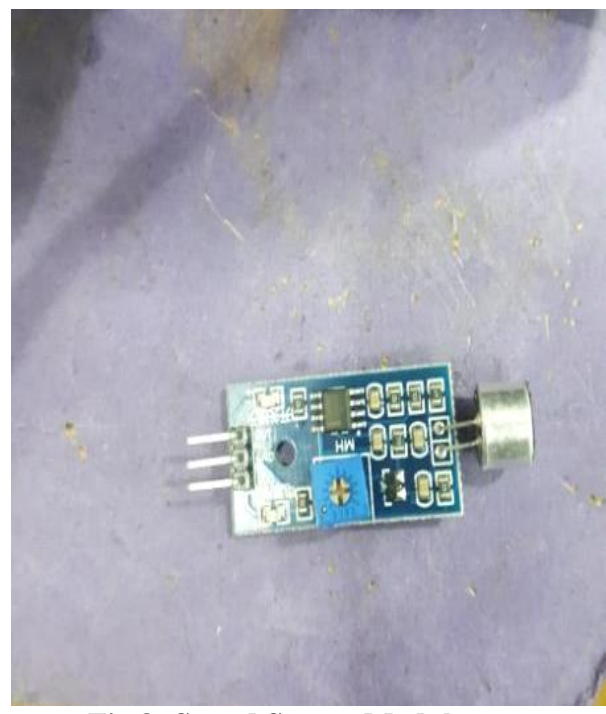

Fig 8: Sound Sensor Module

\section{BUZZER}

A buzzer is a mechanical, electromechanical, magnetic, electromagnetic, electro-acoustic or piezoelectric audio signalling device. A piezo electric buzzer can be driven by an oscillating electronic circuit or other audio signal source. A click, beep or ring can indicate that a button has been pressed.. 


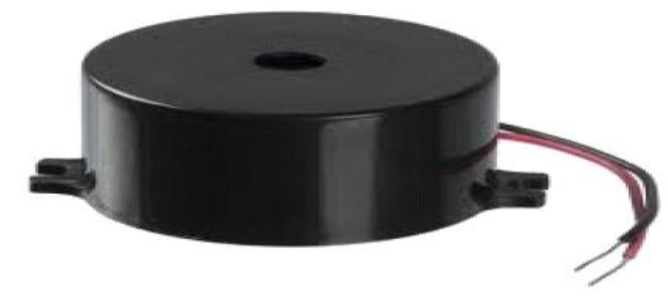

Fig 9: Buzzer

\subsection{Working of Buzzer}

- A buzzer takes some sort of input and emits a sound in response to it.

- $\quad$ They may use various means to produce the sound; everything from metal clappers to electromechanical devices. This can be understood by a simple circuit:

- A buzzer needs to have some way of taking in energy and converting it to acoustic energy.

- Many buzzers are part of a larger circuit and take their power directly from the device's power source. In other cases, however, the buzzer may be battery powered so that it will go off in the event of a mains outage.

- Some devices that provide emergency power have buzzers on them so that the user knows that they are running on backup power and not on mains power.

\subsection{Modern applications}

- Novelty uses

- Judging Panels

- Educational purposes

- Annunciator panels

- $\quad$ Electronic metronomes
- Game showlock-out device

- ovens and other household appliances

- $\quad$ Sporting events such as basketball games

- Electrical alarms

- Joy buzzer- a mechanical buzzer used for pranks.

\section{DHT 11 TEMPERATURE AND HUMIDITY SENSOR}

The DHT11 is a basic, ultra low-cost digital temperature and humidity sensor. It uses a capacitive humidity sensor and a thermistor to measure the surrounding air, and spits out a digital signal on the data pin (no analog input pins needed). It's fairly simple to use, but requires careful timing to grab data..

This DH11 (Temperature \& humidity sensor) has features a calibrated digital signal output with the temperature and humidity sensor complex. Its technology ensures the high reliability and excellent long-term stability. It has excellent quality, fast response, anti-interference ability and high cost performance advantages. Each DHT11 sensor features extremely accurate calibration of humidity calibration chamber. The calibration coefficients stored in the OTP program memory, internal sensors detect signals in the process, we should call these calibration coefficients.

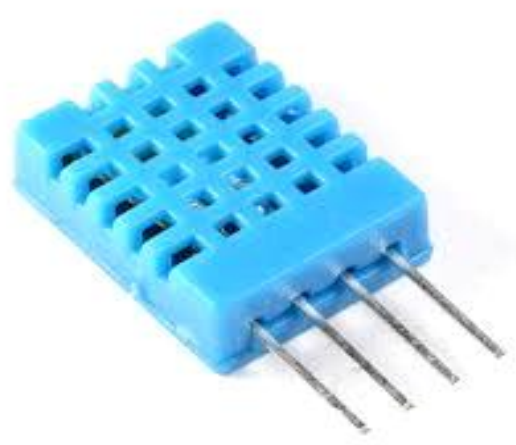

Fig 10: DHT 11 Temperature and Humidity Sensor

Measurement and control of temperature and relative humidity finds applications in numerous areas. These days devices are available which have both temperature and humidity sensors with signal conditioning, ADC, calibration and communication interface all built inside them. The use of such smart sensors greatly simplifies the design and reduces the overall cost. The DHT11 sensor comes in a single row 4pin package and operates from 3.5 to $5.5 \mathrm{~V}$ power supply. It can measure temperature from $0-50{ }^{\circ} \mathrm{C}$ with an accuracy of $\pm 2^{\circ} \mathrm{C}$ and relative humidity ranging from $20-95 \%$ with an accuracy of $\pm 5 \%$. The sensor provides fully calibrated digital outputs for the two measurements. It has got its own proprietary 1-wire protocol, and therefore, the communication between the sensor and a microcontroller is not possible through a direct interface with any of its peripherals. The protocol must be implemented in the firmware of the MCU with precise timing required by the sensor.

\subsection{Technical Details}

- Low cost

- 3 to $5 \mathrm{~V}$ power and $\mathrm{I} / \mathrm{O}$ 
- $\quad 2.5 \mathrm{~mA}$ max current use during conversion (while requesting data)

- Good for $20-80 \%$ humidity readings with $5 \%$ accuracy

- Good for $0-50^{\circ} \mathrm{C}$ temperature readings $\pm 2{ }^{\circ} \mathrm{C}$ accuracy

- No more than $1 \mathrm{~Hz}$ sampling rate (once every second)

- Body size $15.5 \mathrm{~mm}$ x $12 \mathrm{~mm}$ x $5.5 \mathrm{~mm}$

- 4 pins with 0.1 " spacing

\subsection{Hardware Connections}

\section{DTH11 To Xmega 2560}

- $\quad \mathrm{Vcc}---5 \mathrm{v}$

- $\quad$ GND --- GND

- Data Pin --- DIGITAL PIN of Xmega 2560

9. MQ-135 GAS SENSORThe MQ series of gas sensors utilizes a small heater inside with an electro chemical sensor these sensors are sensitive to a range of gasses are used at room temperature. MQ135 alcohol sensor is a Sno2 with a lower conductivity of clean air. When the target explosive gas exists, then the sensor's conductivity increases more increasing more along with the gas concentration rising levels. By using simple electronic circuits, it converts the charge of conductivity to correspond output signal of gas concentration. The MQ135 gas sensor has high sensitivity in ammonia, sulfide, bonze steam, smoke and in other harm full gas. It is low cost and suitable for different applications. A hazardous gas detection apparatus for the family, the environment, suitable for ammonia, aromatic compounds, sulfur, benzene vapor, smoke and other gases harmful gas detection, gas sensitive element test concentration. Air quality sensor is for detecting a wide range of gases, including $\mathrm{NH} 3$, NOx, alcohol, benzene, smoke and CO2. Ideal for use in office or factory, simple drive and monitoring circuit. The MQ-135 gas sensor senses the gases like CO2, ammonia nitrogen, oxygen, alcohols, aromatic compounds, sulfide and smoke. The operating voltage of this gas sensor is from $2.5 \mathrm{~V}$ to $5.0 \mathrm{~V}$. In the atmosphere we can find polluting gases, but the conductivity of gas sensor increases as the concentration of polluting gas increases. MQ-135 gas sensor can be implementation to detect the smoke, benzene, steam and other harmful gases. It has potential to detect different harmful gases. The MQ-135 gas sensor is low cost to purchase.

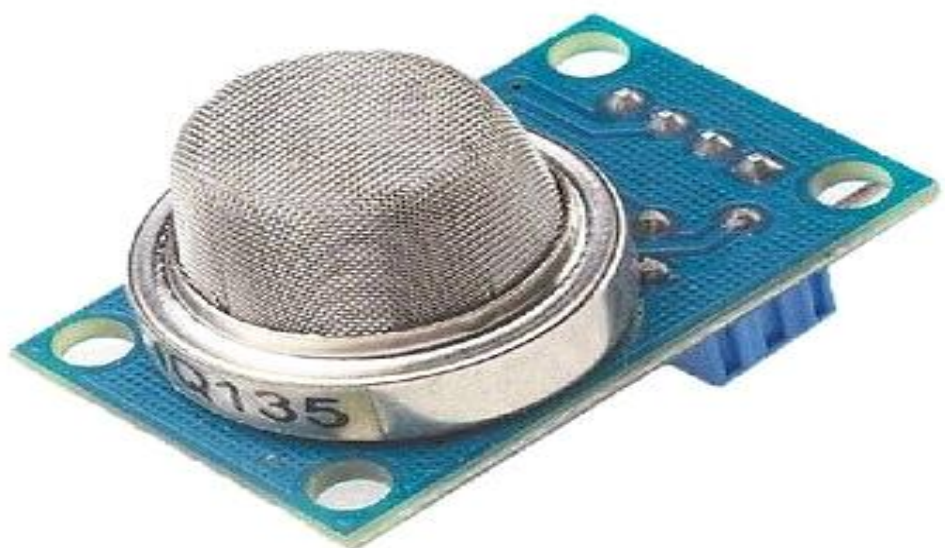

Fig 11:. Mq-135 Gas Sensor

\subsection{Working}

The MQ-135 alcohol sensor consists of a tin dioxide ( $\mathrm{SnO} 2)$, a perspective layer inside aluminum oxide micro tubes (measuring electrodes) and a heating element inside a tubular casing. The end face of the sensor is enclosed by a stainless steel net and the back side holds the connection terminals. Ethyl alcohol present in the breath is oxidized into acetic acid passing through the heat element. With the ethyl alcohol cascade on the tin dioxide sensing layer, the resistance decreases. By using the external load resistance the resistance variation is converted into a suitable voltage variation. The circuit diagram and the connection arrangement of an MQ 135 alcohol is shown below.

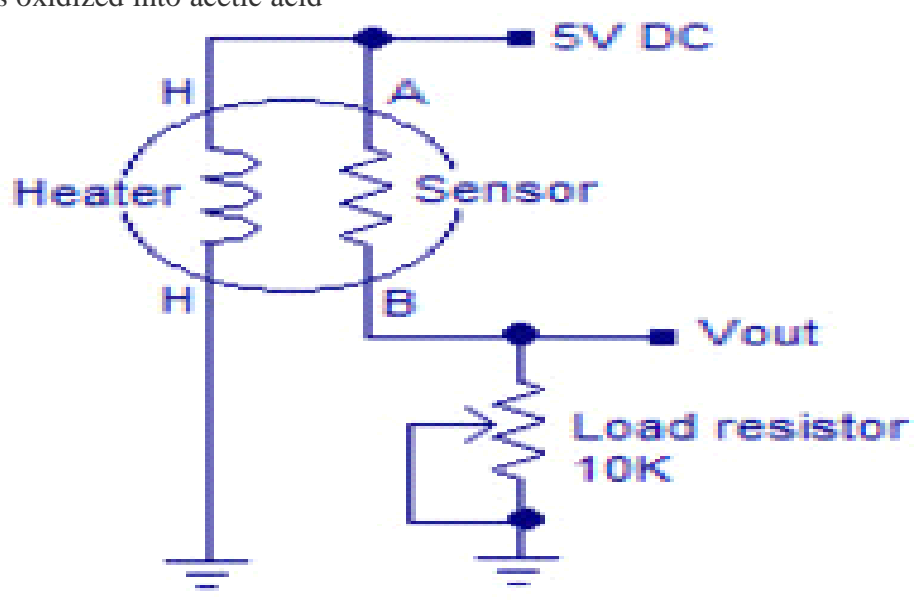




\section{Fig 12 : Working Of MQ-135 Gas Sensor}

\subsection{Characteristics}

- Good sensitivity to harmful gases in wide range

- High sensitive to ammonia sulfide benze

- $\quad$ Long life

- Low cost

- Simple circuitry.

\section{WIFI MODULE}

The Wi-Fi Module is a self-contained SOC with integrated TCP/IP protocol stack that can give any microcontroller access to your Wi-Fi network. The WIFI is capable of either hosting an application or offloading all Wi-Fi networking functions from another application processor. Each WIFI module comes pre-programmed with an AT command set firmware, meaning, you can simply hook this up to your Xmega 2560 device and get about as much Wi-Fi-ability as a WiFi Shield offers (and that's just out of the box)! The WIFI module is an extremely cost effective board with a huge, and ever growing, community. The ESP8266 Wi-Fi module is a self-contained SOC with integrated TCP/IP protocol stack that can give any microcontroller access to your Wi-Fi network. The ESP8266 is capable of either hosting an application or offloading all $\mathrm{Wi}-\mathrm{Fi}$ networking functions from another application processor. ESP8266 module is pre-programmed with an AT command set firmware. This module has a powerful enough on- board processing and storage capability that allows it to be integrated with the sensors and other application specific devices through its GPIOs with minimal development up-front and minimal loading during runtime. It contains a self-calibrated RF allowing it to work under all operating conditions, and requires no external RF parts.

\subsection{ESP8266 Module}

The ESP8266 Wi-Fi module is a self-contained SOC with integrated TCP/IP protocol stack that can give any microcontroller access to your Wi-Fi network. The ESP8266 is capable of either hosting an application or offloading all Wi-Fi networking functions from another application processor. Each ESP8266 module comes pre-programmed with an AT command set firmware]. This module has a powerful enough on-board processing and storage capability that allows it to be integrated with the sensors and other application specific devices through its GPIOs with minimal development up-front and minimal loading during runtime. Its high degree of on-chip integration allows for minimal external circuitry. The ESP8266 Module is not capable of 5-3V logic shifting and will require an external logic level converter. This small module allows microcontrollers to connect to a Wi-Fi network and make simple TCP/IP connections.

This module has a powerful enough on-board processing and storage capability that allows it to be integrated with the sensors and other application specific devices through its GPIOs with minimal development up-front and minimal loading during runtime. Its high degree of on-chip integration allows for minimal external circuitry, including the front-end module, is designed to occupy minimal PCB area. The ESP8266 supports APSD for VoIP applications and Bluetooth co-existence interfaces; it contains a self-calibrated RF allowing it to work under all operating conditions, and requires no external RF parts.There is an almost limitless fountain of information available for the ESP8266, all of which has been provided by amazing community support. In the Documents section below you will find many resources to aid you in using the WIFI, even instructions on how to transforming this module into an IoT (Internet of Things) solution.

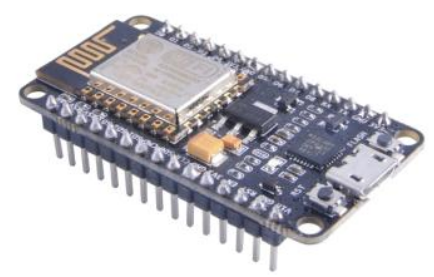

Fig 13: ESP8266 Module

To make everything work, you need to understand what each part does and what the pin out for that part is. I'll start with the ESP8266. As shown in the picture, ESP8266 has 8 pins, 4 in the row of 2. The first pin on the top left is GND. The two pins right from the GND are GPIO 2 and 0. I'm not going to use these pins, as they are not important for the operation. The pin on the top right side is the RX pin and the pin on the lower left is TX. These are the pins for communication. The middle pins on the bottom are CH_PD (chip power-down) and RST (reset). 


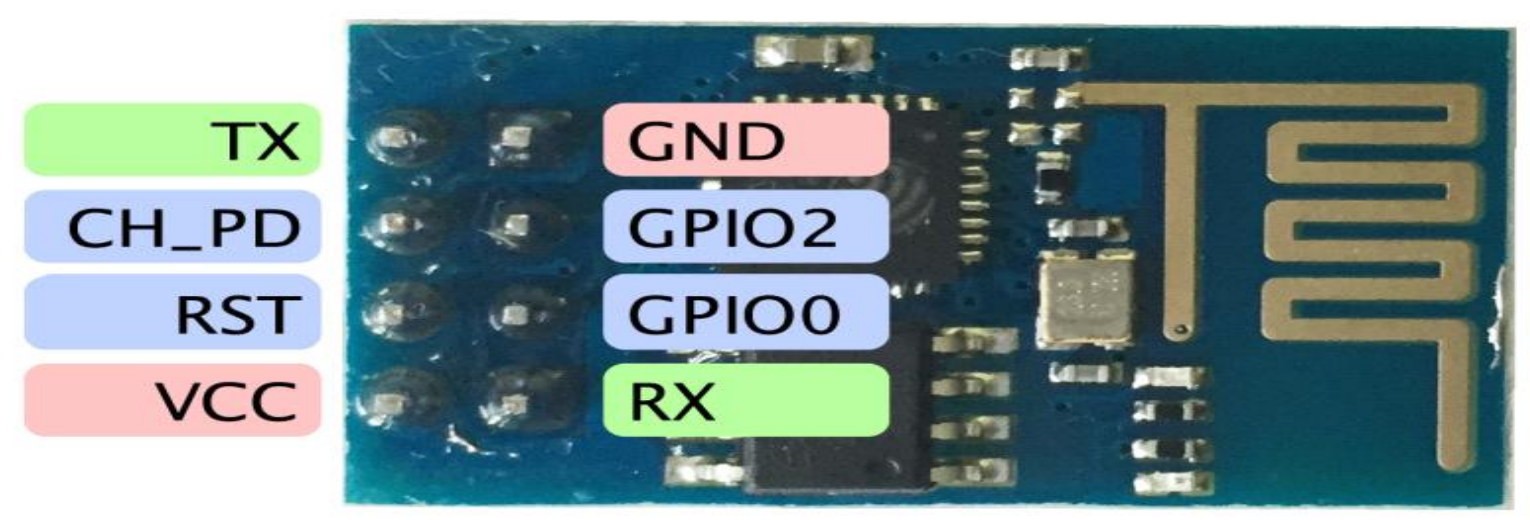

Fig 14: Working of ESP8266 Module

The main thing to remember is, that this device works with $3.3 \mathrm{~V}$; even the RX and TX pins. Xmega 2560 or many USB to serial converters work with $5 \mathrm{~V}$. The solution for this project is in the next step.

\subsection{FEATURES}

- $802.11 \mathrm{~b} / \mathrm{g} / \mathrm{n}$

- Wi-Fi Direct (P2P), soft-AP

- Integrated TCP/IP protocol stack

- Integrated TR switch, balun, LNA, power amplifier and matching network

- Integrated PLLs, regulators, DCXO and power management units

- $\quad+19.5 \mathrm{dBm}$ output power in $802.11 \mathrm{~b}$ mode

- Power down leakage current of $<10 \mathrm{uA}$

- $1 \mathrm{MB}$ Flash Memory

- Integrated low power 32-bit CPU could be used as application processor

- $\quad$ SDIO 1.1 / 2.0, SPI, UART

- $\quad$ STBC, $1 \times 1$ MIMO, $2 \times 1$ MIMO

- A-MPDU \& A-MSDU aggregation \& $0.4 \mathrm{~ms}$ guard interval

- Wake up and transmit packets in $<2 \mathrm{~ms}$

- Standby power consumption of $<1.0 \mathrm{~mW}$ (DTIM3)

\subsection{APPLICATIONS OF THE PROJECT}

- Roadside pollution Monitoring.

- Industrial Perimeter Monitoring.

- Site selection for reference monitoring stations.

- Indoor Air Quality Monitoring.

- Design server using IoT and upload data on that server with date and time.

- To make this data available to the common man.

- To set a danger limit on that server and inform authorities to take future actions for wellbeing.

\section{CONCLUSION}

To implement this need to deploy the sensor devices in the environment for collecting the data and analysis. By deploying sensor devices in the environment, we can bring the environment into real life i.e. it can interact with other objects through the network. Then the collected data and analysis results will be available to the end user through the Wi-Fi.

The smart way to monitor environment and an efficient, low cost embedded system is presented with different models in this paper. In the proposed architecture functions of different modules were discussed. The noise and air pollution monitoring system with Internet of Things (IoT) concept experimentally tested for monitoring two parameters. It also sent the sensor parameters to the cloud (Google Spread Sheets).

This data will be helpful for future analysis and it can be easily shared to other end users. This model can be further expanded to monitor the developing cities and industrial zones for pollution monitoring. To protect the public health from pollution, this model provides an efficient and low cost solution for continuous monitoring of environment.

\section{REFERENCES}

[1] Murty, R.N.; Mainland, G.; Rose, I.; Chowdhury, A.R.; Gosain, A.;Bers, J.; Welsh, M.; CitySense: An UrbanScale Wireless Sensor Network and Testbed, IEEE Conference on Technologies for Homeland Security 2008.,583- 588 .

[2] Barrenetxea, G.; Ingelrest, F.; Schaefer, G.; Vetterli, M.; Couach, O.;Parlange, M. ; SensorScope: Out-of-the- Box Environmental Monitoring,International Conference on Information Processing in SensorNetworks, 2008. IPSN2008. 332 - 343565.

[3] Xu, K.D., Zhang, Y.H., Spiegel, R.J., Fan, Y., Joines, W.T., Liu, Q.H.: Design of a Stub- Loaded RingResonator Slot for Antenna Applications. Antennas and Propagation, IEEE Transactions on (2015), vol.63, no.2, pp.517, 524.

[4] Sim, C.-Y.-D., Shih, Y.-K., Chang, M.-H.: Compact slot antenna for wireless local area network 2.4/5.2/5.8 GHz applications. Microwaves, Antennas \& Propagation, IET (2015), vol.9, no.6, pp.495, 501.

[5] Yeom, I., Kim, J.M., Jung, C.W.: Dual-band slot-coupled patch antenna with broad bandwidth and high directivity for WLAN access point, Electronics Letters (2014), vol.50, no.10, pp.726,728.

[6] Huang, H.-F., Hu, Y., A Compact Dual-Band Printed Monopole Antenna for WiMax /WLAN Applications. 
Progress in Electromagnetics Research Letters (2014), Vol. 49, 91- 97.

[7] Joo, D.Y and Kim, J.K.: Creative \& active convergence model of IoT, Korea Institute for Industrial Economics \& Trade, Korea (2014).

[8] Bagula, A., Castelli, L and Zennaro, M.: On the design of smart parking networks in the smart cities: An optimal sensor placement model, Sensors, (2015), Vol.15, No.7, pp.15443-15467.

[9] Xu Cheng, Minghui Zhang, Fuquan Sun, "Architecture of internet of things and its key technology integration based-on RFID," in Fifth International Symposium on Computational Intelligence and Design, pp. 294-297, 2012.

[10] I.Akyildiz and J.Jornet, "THE INTERNET OF NANOTHINGS," IEEE Wireless Communications, Volume: 17 Issue: 6, 2010, pp. 58-63
[11] B.B.P. Rao, P.Saluia, N.Sharma, A.Mittal, S.V.Sharma, "Cloud computing for Internet of Things \& sensing based applications," in Sensing Technology (ICST), 2012 Sixth International Conference, IEEE

[12] Sohraby, K., Minoli, D., Znati, T. "Wireless sensor networks: technology, protocols, and applications", John Wiley and Sons, 2007 ISBN 978-0-471-74300-2, pp. 1518

[13] X. Xiaohui, "Study on Security Problems and Key Technologies of The Internet of Things," Computational and Information Sciences (ICCIS), 2013, pp. 407-410.

[14] I.Akyildiz and J.Jornet, "THE INTERNET OF NANOTHINGS," IEEE Wireless Communications, Volume: 17 Issue: 6, 2010, pp. 58-63 